\title{
Towards Flexible Process Automation
} An Approach for Flexible Service Robot Adaptation and Allocation

\author{
Matthias Rollenhagen \\ Institute of Computer Science, \\ University of Applied Sciences UIm, \\ Prittwitzstrasse 10, 89075 Ulm, \\ Germany \\ rollenhagen@hs-ulm.de
}

\author{
Matthias Lutz \\ Institute of Computer Science, \\ University of Applied Sciences UIm, \\ Prittwitzstrasse 10, 89075 Ulm, \\ Germany \\ lutz@hs-ulm.de
}

\author{
Nayabrasul Shaik \\ Institute of Computer Science, \\ University of Applied Sciences UIm, \\ Prittwitzstrasse 10, 89075 Ulm, \\ Germany \\ shaik@hs-ulm.de
}

\section{Kevin Andrews \\ Institute of Databases and \\ Information Systems, UIm Universty, Information Systems, Ulm \\ Sebastian Steinau \\ Institute of Databases and \\ James-Franck-Ring, $89081 \mathrm{Ulm}$, Germany \\ kevin.andrews@uni-ulm.de

\author{
James-Franck-Ring, $89081 \mathrm{UIm}$, \\ Germany \\ Germany
} \\ sebastian.steinau@uni-ulm.de manfred.reichert@uni-ulm.de \\ Manfred Reichert
Institute of Databases and \\ Institute of Databases and
rmation Systems, Ulm University, \\ James-Franck-Ring, 89081 Ulm,}

\author{
Christian Schlegel \\ Institute of Computer Science, \\ University of Applied Sciences \\ Ulm, Prittwitzstrasse 10, 89075 Ulm, \\ Germany \\ schlegel@hs-ulm.de
}

\begin{abstract}
The dynamic of today's markets demand automation systems that are flexible enough for being adapted to rapid requirement changes. The presented work describes how to make robotic systems more flexible in order to cope with changing processes, products, and environments. We propose flexibilizing service robot task modeling and assignment by coupling them with process management systems and utilize them similarly to human workers. Additionally, a variability management workflow is presented, for not fixing systems at design-time. Instead, variability is left open intentionally and reduced gradually. The combination of these two approaches enables users (e.g. workers) to adapt their robot systems to requirement changes by userfriendly tools and provides flexible service robot allocation.
\end{abstract}

\section{CCS Concepts}

-Computer Systems Organization $\rightarrow$ External Interfaces for Robotics; Robotic Control;

\section{Keywords}

Permission to make digital or hard copies of all or part of this work for personal or classroom use is granted without fee provided that copies are not made or distributed for profit or commercial advantage and that copies bear this notice and the full citation on the first page. Copyrights for components of this work owned by others than the author(s) must be honored. Abstracting with credit is permitted. To copy otherwise, or republish, to post on servers or to redistribute to lists, requires prior specific permission and/or a fee. Request permissions from Permissions@acm.org.

ISCSIC 2019, September 25-27, 2019, Amsterdam, Netherlands (C) 2019 Copyright is held by the owner/author(s).

ACM ISBN 978-1-4503-7661-7/19/09.

https://doi.org/10.1145/3386164.3387292
Service Robot; Software Systems; Variability Management

\section{INTRODUCTION}

Due to shorter product life cycles, adaptation to rapid market changes is one of the key challenges companies nowadays are confronted with. For industry, this dynamics poses a structural change on all levels of production, from mass-production to massindividualization. This requires industry to implement highly flexible and rapidly adaptable processes. Especially small- and medium-sized companies are confronted with a number of new challenges:

(1) Conventional static automation machinery can only be used for a specific setting, not providing the required flexibility for rapidly changing processes or products. Adaptations typically require the involvement of external experts (integrators), which is very expensive and time-consuming. (2) The unpredictability of requirements, combined with present automation inflexibility, makes it hard to invest in automation systems because companies are not able to cope with the short payback periods. (3) Seasonal or spontaneous temporal fluctuations cause frequent under- or over-utilization of automation systems, due to fixed dimensioning.

Today, due to these challenges, supporting processes, which are not directly value-adding (e.g. logistics), are carried out manually to a large degree. The advantage of manual work is the flexible allocation of humans to different processes, according to their load. For example, return shipments typically arrive at a specific time every day. After they are processed the employees can be assigned to a different process currently exhibiting a higher load. However, if a sorting machine for return shipments is used instead of human workers, this machine stays idle until the delivery of return shipments on the next day. Employing human work enables companies to reach a high utilization of working resources. But 
the major downside is the expense of human labor.

In contrast to conventional automation machinery, service robots generally can be used flexibly according to location and task. Thus, service robots offer the potential of being just as flexibly allocated as human workers. If it is possible to assign service robots to different tasks, dependent on the demand, their idle times will drastically reduce and payback periods can be extended. Therefore, service robots need to be easily adaptable to new processes, products, and environments on short notice. Taking one step towards achieving this goal, this paper provides the following contributions:

- Easy adaptation is enabled by integrating robot users into the development process using domain-specific tools to bind intentionally left open system-variability.

- Dynamic task assignment is enabled by coupling service robots with process management systems using variability during development and run-time.

In Section 3, a variability management workflow is described to handle the required variability by describing which role performs which task during development or adaptation. Section 4 describes how variability can be made accessible from a system-structural point of view. This is done by describing the modular connection between robot systems and process management systems. Section 5 provides a proof of concept using an example from the intralogistics domain.

\section{RELATED WORK}

The larger context of this work is defined by production research, requesting modular, scalable, reconfigurable, and flexible automation systems [12]. Different paradigms and methods were proposed for achieving this goal (e.g. holonic manufacturing systems [19], reconfigurable manufacturing systems [26], and evolvable production systems [18]). This paper presents an approach describing how the shared requirements can be realized with a service robot system.

Correspondingly, we define adaptable service robotic systems as "designed at the outset for rapid change in structure, as well as in hardware and software components, in order to quickly adjust [...] capacity and functionality [...] in response to sudden changes in market or in regulatory requirements." [26]

Adaptability based on architectural design is not new to robotics. Conversely, there is an ongoing discussion about adequate software system architectures to support fast development and adaptation. A study [1] found that, in recent years, one major focus of research in the field of software engineering for robotic systems was on architecture-driven reconfiguration, to adapt robots' components and behavior to changing requirements of its environment. Most of the proposed solutions to this problem rest on model-driven methods. But also service-oriented and component-based architectures are used widely. In most cases, these three development methods are utilized independently. Pons [5] predicts a promising combination of these three development methods in robotics, but also states a lack of systems exploiting this combination. This combination is utilized in the presented work using SmartSoft [8, 24].

Adaptability achieved by enabling non-experts to program robots to meet their specific needs is also an active field of research, that is divided into two main categories. Programming robots by demonstration $[4,2]$ is the most popular technique in the literature for easily teaching robots new behavior. The other wide spread approach is visual programming, which allows to specify program elements graphically rather than textually. A great variety of systems for visually programming mobile general-purpose robots was proposed. For this reason, only a small selection of developments can be mentioned: ROS Commander [11], RoboFlow [21] and Interaction Composer [7] provide a flowbased visual programming for robot actions, using boxes as representations of functions, procedures or states and lines for transitions between them. Code3 [13] provides a graphical highlevel programming interface for control flow logic and integrates components for simplifying perceptions and manipulations. These methods are typically developed for education or relatively simple scenarios with small processes, static environment and no robotrobot-interaction. The method presented in this paper differs from these approaches, since it focuses on the usage in industrial processes in large environments with a heterogeneous robot fleet. This is taken into account by utilizing visual programming on task level and supporting separation of roles, which is a decisive characteristic of expanding markets and a robotic business ecosystem [6].

One key idea behind the presented approach is the coupling of a robot fleet with a higher-level management system. In $[9,10,16]$ the authors integrated their system (SkiROS) into a manufacturing execution system to autonomously perform a logistics process. This approach mainly focuses on the planning/optimization of task execution and the utilized context knowledge representation. This method is very close to the presented work, but the integration in the cited works is not generalized. The presented work focuses on the flexibility of processes, and, hence, goes further in describing the structures required for a general coupling of a robotic fleet with a management system. Furthermore, we aim to utilize humans and robots equally by the management system.

\section{VARIABILITY MANAGEMENT}

In previous works [3, 17], we showed that a robotic software system should not be fixed at design-time, but should contain purposefully left open variability, which is gradually reduced across the development phases using the specific knowledge of the different roles about the area of variability. Beside two domain-specific languages a variability management workflow (Figure 1), covering the whole development process, was presented. The original workflow is based on four phases (design, implementation, integration and run-time), which are performed by specific roles. Transitions between phases represent interfaces between roles for passing artifacts (e.g. behavior models, context models). This allows stepwise model refinement, where each role individually contributes to the overall system according to its expertise. The different roles of the workflow can collaborate in a robotic business ecosystem [20], sharing risks and benefiting from their dedicated expertise. Developing an application establishes a relation between the independent stakeholders and involves them in the workflow by contributing their work to one of the phases.

In the following, this approach to variability management is extended by adding the idea of system adaptation by users through domain-specific and model-driven software tools to provide more flexibility to system owners.

Due to the market behavior, robotic systems can no longer be considered as a one time investment for fulfilling one single task, but rather have to be adapted to changing tasks and contexts putting the system under construction regularly. Consequently, the 


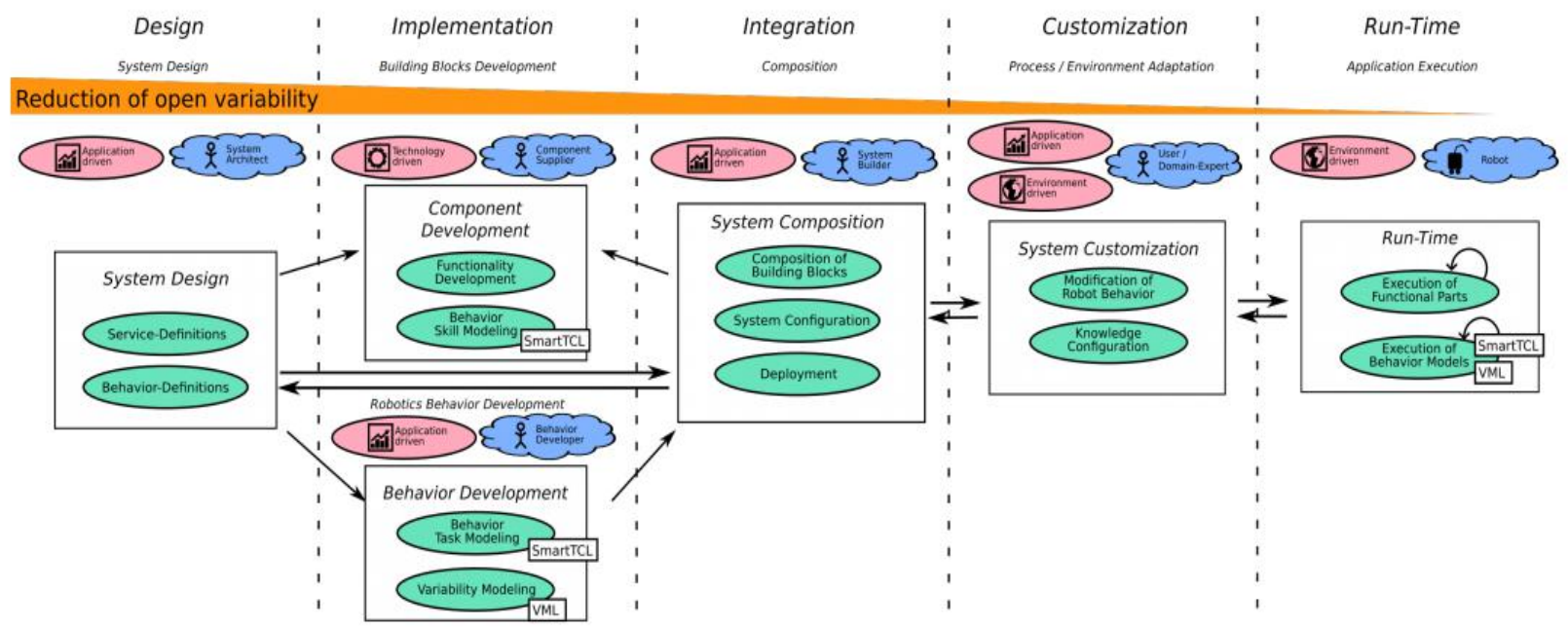

Figure 1: The proposed variability management workflow enables the involved roles to intentionally leave open variability and bind it later, by the role with best knowledge about the individual variation points.

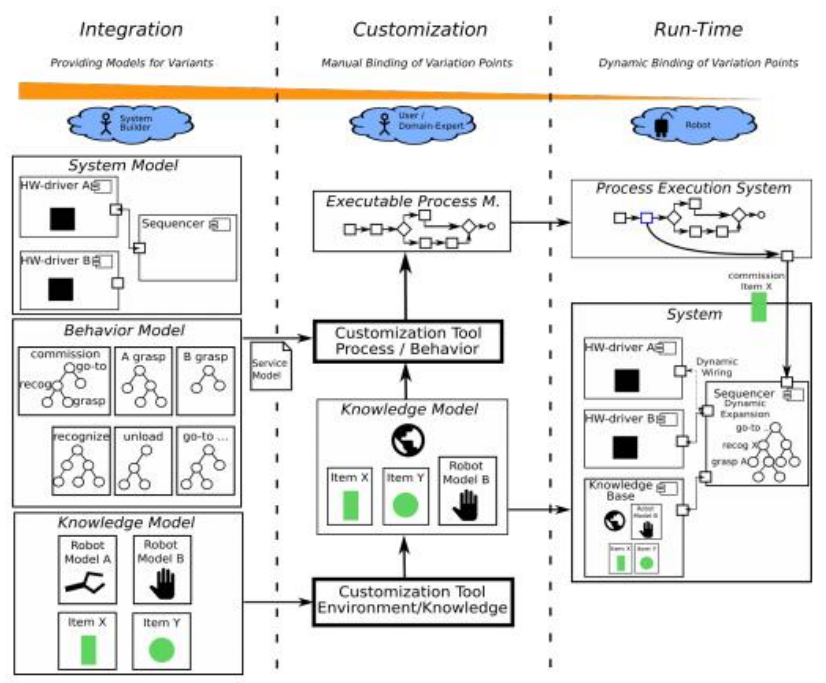

Figure 2: Excerpt of the variability management workflow that depicts how customization of the different models results in system adaptation.

development workflow (Figure 1) is processed repeatedly, depending on the requirements' volatility. But repeatedly performing the full workflow while involving all roles into every adaptation keeps companies inflexible, since doing so is inefficient in terms of time and costs. For this reason, we extended the workflow by adding a phase for customizing the robot system to the current situation.

During this customization phase, the system is adapted to the company's current individual requirements, heavily depending on the executed process (application-driven) and the surrounding conditions (environment-driven). For this reason, the role performing the customization needs to be a person who is responsible for the process performed by the system. Consequently, this phase is assigned to the role of companies' domain expert, which can be process engineers or machine operators (system users).

The previous roles are enabled to leave open variability that can be bound by domain experts using domain-specific tools during customization, or the robot during run-time. This relieves robotic experts from gaining too deep domain and environment knowledge, since this can be introduced by the domain experts, who, in turn, are relieved from gaining deep robotic knowledge. Additionally, the reuse of building blocks is enabled in a wider range of applications. The rest of this section describes how this separation of roles can be achieved by constructing systems from building blocks and using domain-specific tools for customization. The description closely follows Figure 2 from Integration to RunTime.

During Integration the system builder develops models that include the different execution variants of a process. In case of the system model, this means there will be several components solving the same problem in a different manner, like a driver component for different robot end effectors. The behavior models are responsible for the orchestration of the components and provide different modular and primitive robot skills (e.g. go-to, grasp). These skills are composed to behavior models of process building blocks (e.g. commission). Since service robots perform physical manipulations to real objects, their behavior bases in great parts on the knowledge about these objects and their environment. As an example, the object recognition component needs to be configured to the characteristics of the searched item (X or Y). But the required knowledge can only be provided during run-time, as the robots work in a dynamic environment. Consequently, behavior models provide variation points for context knowledge, which stay unbound until run-time.

Starting with Customization the behavior and knowledge models are handed to the domain-expert, who loads them into to the appropriate domain-specific tools. The single knowledge models are structured by adding relations and custom information using the environment modeling tool. The resulting knowledge is forwarded to the process modeling tool, which enables the user to compose complex processes from available process building blocks (PBB). This tool can guide the user by requesting the required $\mathrm{PBB}$ parameters and provide valid options from the knowledge model. For example, the tool can provide all available stations, that can be used by a robot to deliver a commissioned order. Furthermore, the domain-expert adapts the hardware to current process requirements. Without deep robotic knowledge the domain-expert will only be able to perform minor changes (e.g. 
gripper) with modular hardware interfaces.

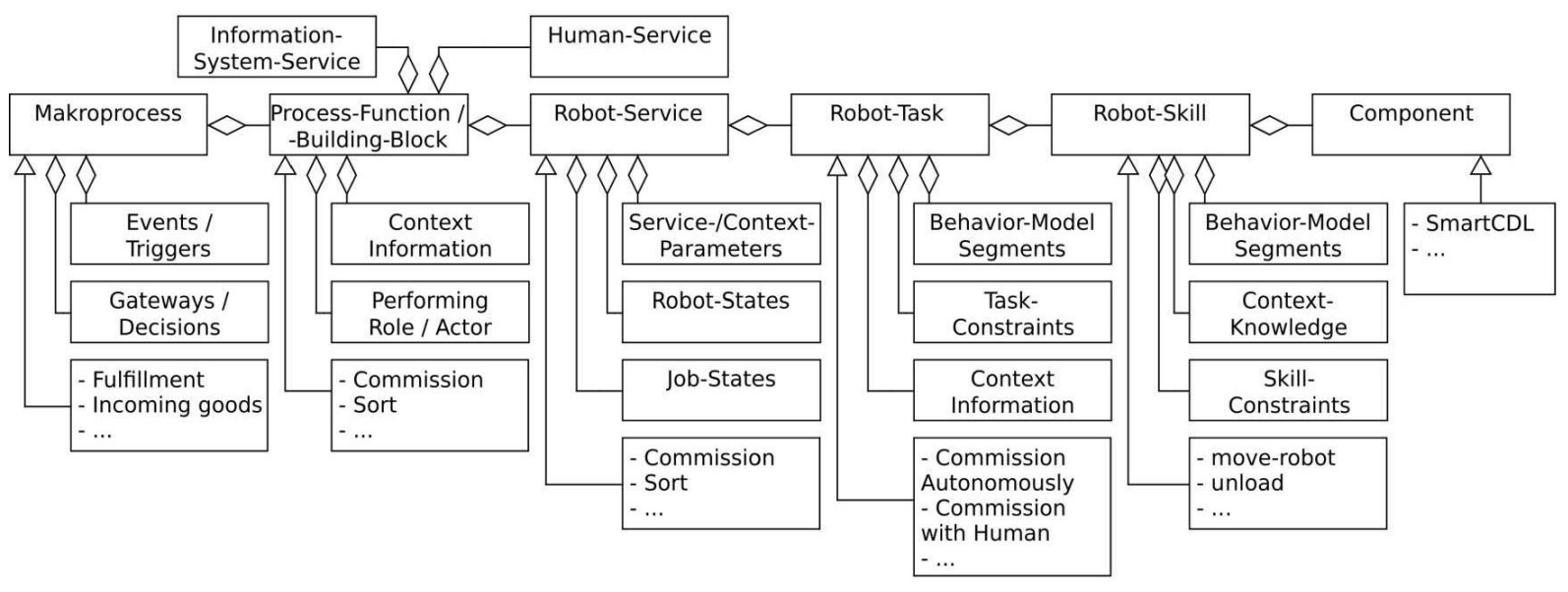

Figure 3: Modular coupling of process modeling and robotic task execution.

For putting the system into Run-Time the behavior and knowledge models are transferred to the robots and the executable process model is imported to a process execution system (MES, ERP, WMS etc.). In Figure 2, the process execution system commands the robot to perform a commissioning PBB for picking an item of type $\mathrm{X}$. The still open variation points are now dynamically bound based on order information and context knowledge.

Binding variability by the role with deepest knowledge about the particular variation point relieves all involved roles from gaining deep knowledge beyond their responsibilities. Furthermore, the modular approach enables system owners to adapt their systems (e.g. implementing a different process by rearranging the available services) without involving robotic experts every time.

\section{PROCESS MANAGEMENT COUPLING}

In SOA-based process management systems (PMS) processes are implemented by orchestrating single services of different systems. The presented method uses the same approach: Working resources (humans, robots) provide services (manual activities) to a PMS. These services lead to an overall result by integrating them into the greater context of a process. Robots and human workers are interchangeable, as they may provide the same services. This combined integration enables an overview of the currently available working resources and the open activities. Having this knowledge and knowing the capacity (speed, strength etc.) of the service providing resource, PMSs can optimize the execution of the current situation and reduce idle times.

To enable this kind of coupling, this section describes an approach connecting abstract process definitions (e.g. in BPMN) to the robot system execution level. The modular approach of this method allows process modelers to construct new processes by composing them from $\mathrm{PBBs}$ and linking the required context knowledge to them. In case of requirement-changes the context knowledge can be remodeled and single missing building blocks can easily be exchanged on all abstraction levels.

The description follows the structure of the depicted approach (Figure 3) from macroprocesses to components.

The entirety of all Tasks within a company consists of a number of defined macroprocesses. The whole intralogistics for instance, is composable by the six macroprocesses incoming goods, storage, line supply, fulfillment sorting and outgoing goods [15]. In a concrete implementation within a company, these macroprocesses can be defined in a process model consisting of events, decisions and process functions.

Each process function represents an activity within a macroprocess performed by an actor. In traditional PMSs, actors can be humans or external information systems. As service robots should perform the same work as human workers (physical manipulations), they are represented as regular actors, with assigned roles. These roles are the PMS's internal representation of the robot-services. By knowing, which service is required for the execution of a process function, it is possible to dynamically assign it to a specific actor. For example, only robots equipped with a manipulator can perform the picking part of a commissioning activity.

Process functions are also called process building blocks to highlight their composability. In intralogistics, all macroprocesses can be composed of overall 14 PBBs [25]. Nine of them (transportation, collecting, distribution, commissioning sorting, unpacking, packing labeling, checking) constitute a physical manipulation of goods, and, hence are doable by service robots. Every PBB poses one process function, even, if several actors are involved. For instance, by composing the PBBs unpacking, checking sorting, and transportation, an incoming-goodsmacroprocess can be implemented. The composability of the PBBs is realized by domain-specific unified interfaces and internal variation points, that are bound with context information during process modeling (Customization Phase, see Figure 2). In the incoming-goods-example, the station IDs, where the goods should be delivered to, are such open context variation points.

Services are the transition between PMS and robot system. Services are used by PMSs for the execution of a process function. A process function binds zero (if humans perform the activity) one, or several robot services. For a commissioning function, a picker and a transporter are required. For this reason, the function is divided into several services. The system can also use both services of the same actor (e.g. a robot with a storage area for the picked items).

A robot-service can be expressed by several alternative implementations named robot-tasks. These variants perform the same service (what), but in a different manner (how). Consequently, services represent the interface description between 
process-model and -execution. Tasks, in contrast, implement concrete execution variants of the overlying service. The decision

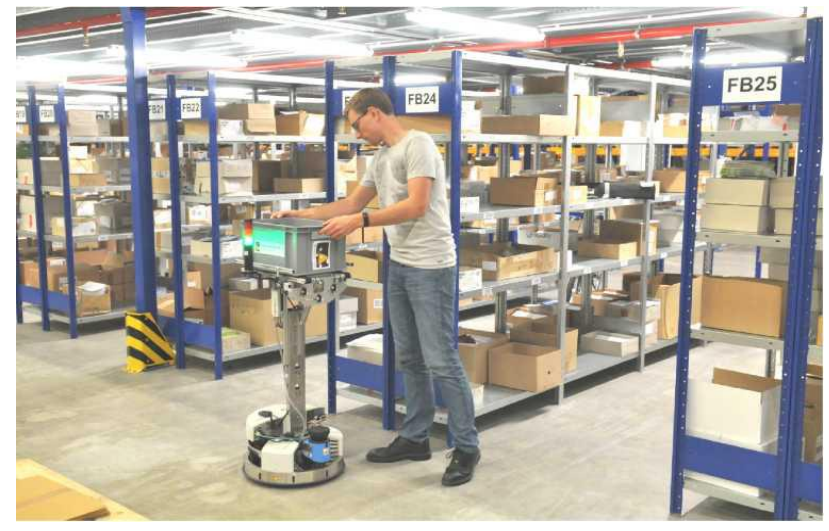

Figure 4: Human-robot-collaborative commissioning with an assistive transportation robot.

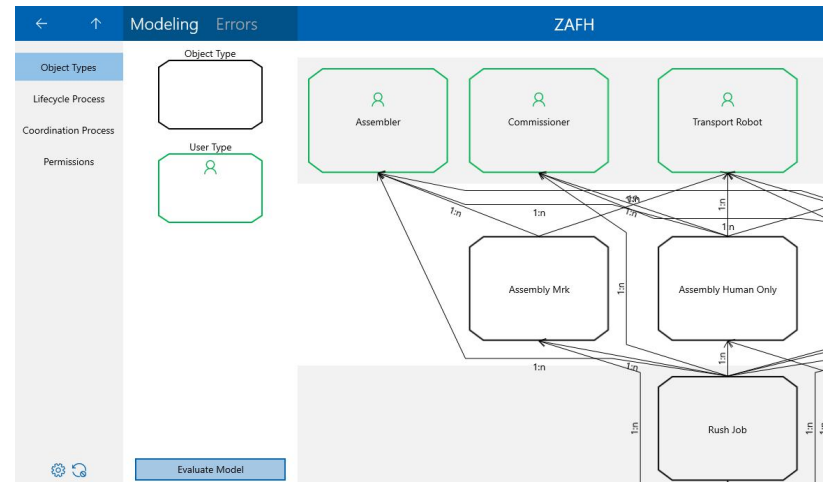

Figure 5: Process modeling interface of PHILharmonicFlows, used to modularly compose processes.

how the service should be executed, meaning which task is chosen, is decided by the PMS during run-time and communicated to the robot via the service parameters. If no picking-robot is available, the service of a human worker is bound. In parallel the service of a transportation-robot is bound. But the robot is instructed to use its person-following-skill, for this job, instead of its robot-robotinteraction-skill.

Robot-tasks, in turn, are composed of a sequential or parallel execution of robot-skills. For instance, the task "transport" sequentially uses the skills "move-robot", for approaching the delivery station, and "unload" for docking to the station and hand over the small-load-carrier (SLC). Robot skills abstract the usage of software components and provide basic robot abilities. Variability on skill-level is expressed by selecting and configuring components. Performing the hand over of a SLC to a human requires a different behavior than to an automatic station. In both cases, the same skill (unload) is used, but different components are involved. The execution variant is chosen during run-time by linking the provided service parameters (e.g. station ID) and context knowledge (e.g. station type). This shows the necessity of context information for correct and variable task-execution.

Furthermore, the PMS can define constraints on how tasks and skills shall be executed. These variabilities are bound via the service parameters. A rush-job, for instance, increases the robots speed and changes its navigation strategy.

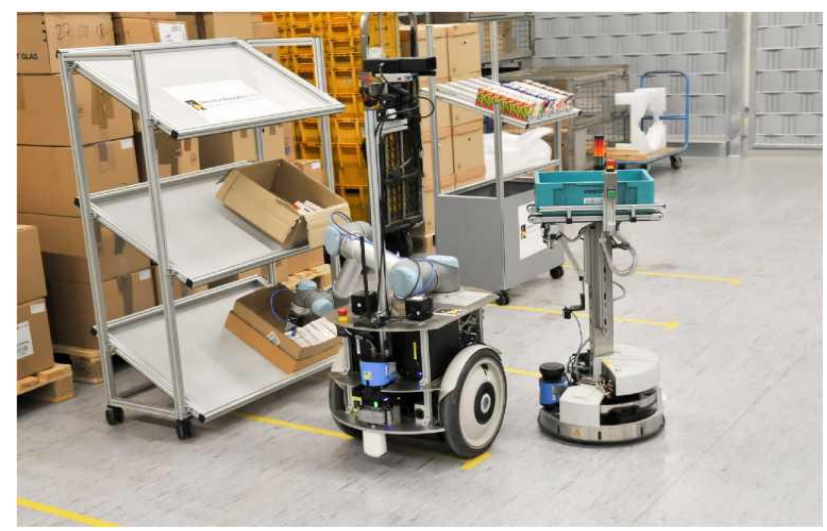

Figure 6: Collaborative robotic commissioning, autonomously picking pharmaceutical packages from cardboard boxes in an industrial environment.

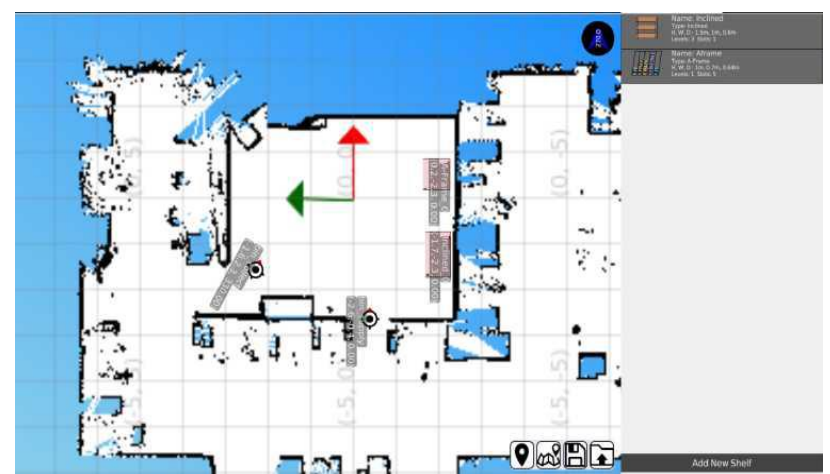

Figure 7: Prototype of domain-specific customization tool for modeling intralogistic environments. The GUI shows a map with two placed shelfs.

\section{INTRALOGISTICS APPLICATION}

The application illustrates an intralogistics fulfillment process. Goods are commissioned from a warehouse, and depending on the ordered item type and resource availability, the order picking is performed autonomously by robots (Robotino 3 for transportation and an UR5 manipulator equipped robot for picking, Figure 6) or by an human-robot-collaboration (Robotino 3 as assistive transportation-system, following the human picker through the warehouse, Figure 4). Videos showing the execution of the complete application are available on YouTube $[22,23]$.

The process is modeled and managed by the PMS PHILharmonicFlows [14]. PHILharmonicFlows receives orders from a mocked warehouse management system and creates process instances. Within the graphical modeling environment, processes are composed from a number of process functions (Figure 5). The available robot services (go-to, deliver-slc, commission, etc.) are a predefined subset of these functions. PHILharmonicFlows requests the context information from the fleet management system and provides it to the user, for process function configuration (e.g. delivery station for picked orders). During run-time PHILharmonicFlows situationally decides which resource the current order should be assigned to and which execution variant (task) shall be chosen, i.e. autonomous or human-robot-collaborative.

To illustrate the short-term process adaptability the scenario shows how an additional step is introduced into the process: Due to a special promotion every order receives a merchandise item. This is done using the PHILharmonicFlows modeling interface to add a 
process function to the process model. Due to market dynamics, the industrial layout regularly changed. To adapt the robots to new contexts, a graphical modeling tool was developed (Figure 7). This tool enables the system owner to model the domain-specific warehouse context and distribute it to the involved systems.

Using the described approach enables adaptation of the process and context without expert knowledge within short periods of time. In the following section the system architecture, for realizing the concepts outlined above, is described.

\subsection{System Architecture}

The fleet management system (Fig. 8) receives the information of the context modeling and PMS, manages the information and forwards it to the involved robots. The job dispatcher can choose a robot for execution on the basis of their states and the provided services. Robots regularly send status information to the fleet management. This information is handled by the principle of subsidiarity: If there is some process-related information (e.g. an error which is not solvable by the fleet management) the information is forwarded to the PMS, which has additional possibilities for solving problems (e.g. sending a human to the robot).

Listing 1: JSON-implementation of a job assignment

\{"type": "create",

"obj-type": "job",

"obj-list" : [\{

"id": 25,

"type": "commission",

"robots":

[\{"robot-id": 2, "task": "transport-robot"\},

\{"robot-id": 88, "task": "pick"\}],

"source-id": 3,

"destination-id" : 12 ,

"constraints":["fast/energy-efficient" $]\}]\}$

Listing 1 shows the JSON-implementation for assigning a commissioning job to the robot fleet. This example illustrates many of the described concepts: (1) Several actors are involved in one job (robot 2 and 88). (2) Each actor plays a part of the job, by performing a service (transport, pick). (3) Services provide execution variants by representing abstract activities, which are implemented by concrete tasks. On the service level, behavior models are dynamically composed of building blocks (task, skill). In Figure 8 this is indicated by the green and blue process functions within the PMS, which are found again as task models on the robot level. As transportation can be performed for a human or a robot, the service variability is bound by defining the task (transport-robot). (4) During customization, the context is modeled and used to bind variability of the process functions (station IDs of source and destination). In Figure 8 this is again indicated by the colors red and light blue, which can be found in the context modeler and the skill behavior model. In the skill behavior model, they represent variation points on how to dock to a station, depending on their types, i.e. manual or automatic. (5) On the process level, constraints for the execution of tasks or skills can be defined (transporting fast or energy-efficient).

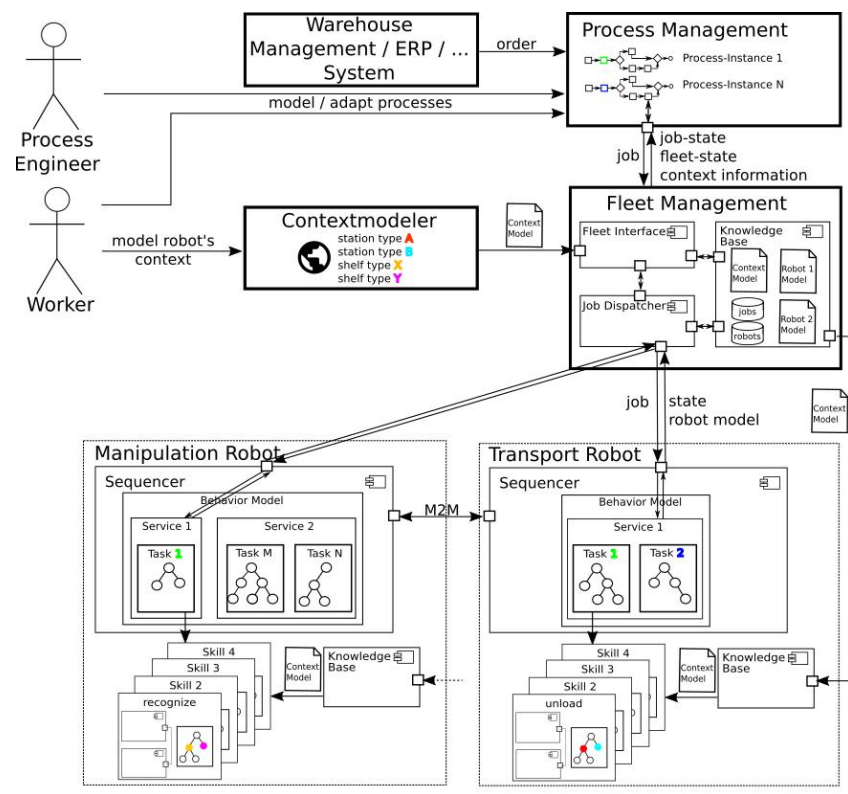

Figure 8: Implemented system architecture for modeling and executing robotic processes using variation points and context knowledge.

\section{CONCLUSION}

The presented work proposes an approach, how companies can cope with the growing requirements concerning flexibility in industrial processes, by means of flexible service robot software systems. To achieve this flexibility, service robots should no longer be regarded as traditional automation systems, but rather being assignable to different processes similarly to human workers and adaptable to new processes, products, and environments on short-notice.

Flexible allocation is achieved by coupling service robots with PMSs. This raises a need for connecting process modeling and robotic task execution. We provide a solution for this by defining the necessary abstraction levels between process definition and robot software component level. This approach allows utilizing robots and human workers interchangeably by PMSs, which enables companies to assign jobs situationally according to resource availability and process load. This reduces the amount of required working resources and allows faster payback of investments.

The extended variability management workflow separates the responsibilities within developments and adaptations between the roles. Now system users are involved in the workflow to adapt systems and bind variability on their own supported by domainspecific tools. Consequently, if there are process changes that do not require new execution variants, adaptations can be performed by the companies without involving expensive developers or integrators, by using process- and context-modeling. The proof of concept shows that these adaptations are possible even during the robots' run-time. Additionally, unbound variability on the different abstraction levels enables the reuse of modular building blocks in different systems, processes, and environments.

All this constitutes one step towards companies being adaptable to dynamic market behavior. Therefore, they are able to spread their payback periods over a longer period of time with multiple contracts and products. The same applies to temporal fluctuation, as service robots can be assigned to different tasks during times of 
under-utilization. Consequently, automation becomes affordable to small- and medium-sized enterprises. New business models, like leasing of service robots and selling off-the-shelf building blocks within a robotic business ecosystem, become possible.

\section{ACKNOWLEDGMENTS}

Supported by "EFRE Program Baden-Württemberg 2014-2020" (ZAFH Intralogistik) and BMBF (LogiRob 01IS16008B).

\section{REFERENCES}

[1] A. Ahmad and M. A. Babar. Software architectures for robotic systems: A systematic mapping study. Journal of Systems and Software, 122:16 - 39, 2016.

[2] A. Billard, S. Calinon, R. Dillmann, and S. Schaal. Robot Programming by Demonstration, pages 1371-1394. Springer, Berlin, Heidelberg, 2008.

[3] A. Lotz, J. F. Ingles-Romero, D. Stampfer, M. Lutz, C. Vicente-Chicote, and C. Schlegel. Towards a stepwise variability management process for complex systems: A robotics perspective. Int. J. Inf. Syst. Model. Des., 5(3):55-74, July 2014.

[4] B. D. Argali, S. Chernova, M. Veloso, and B. Browning. A survey of robot learning from demonstration. Robot. Auton. Syst., 57, 2009.

[5] C. Pons, C. Perez, R. Giandini, and G. Baum. A modeldriving approach to constructing robotic systems. Journal of Computer Science and Technology, 14(1): 1-8, 2014.

[6] C. Schlegel, A. Steck, and A. Lotz. Robotic software systems: From code-driven to model-driven software development. In A. Dutta, editor, Robotic Systems, chapter 23. IntechOpen, Rijeka, 2012.

[7] D. F. Glas, T. Kanda, and H. Ishiguro. Human-robot interaction design using interaction composer: Eight years of lessons learned. In The Eleventh ACM /IEEE International Conference on Human Robot Interaction, HRI '16, pages 303-310, Piscataway, NJ, USA, 2016. IEEE Press.

[8] D. Stampfer, A. Lotz, M. Lutz, and C. Schlegel. The SmartMDSD toolchain: An integrated MDSD workflow and integrated development environment (IDE) for robotics software. Journal of Software Engineering for Robotics (JOSER), 7(1):3-19, 2014.

[9] F. Rovida and V. Krüger. Design and development of a software architecture for autonomous mobile manipulators in industrial environments. In 2015 IEEE International Conference on Industrial Technology (ICIT), pages 32883295, March 2015.

[10] F. Rovida, V. Krüger, L. Nalpantidis, A. Charzoule, A. Lasnier, R. Petrick, M. Crosby, C. Toscano, and G. Veiga. A cyber-physical systems approach for controlling autonomous mobile manipulators. In Advances in Cooperative Robotics, pages 169-177. World Scientific Publishing, 2017.

[11] H. Nguyen, M. T. Ciocarlie, K. Hsiao, mdC. C. Kemp. Ros commander (rosco): Behavior creation for home robots. 2013 IEEE International Conference on Robotics and Automation, pages $467-474,2013$.
[12] J. Barbosa, P. Leitão, E. Adam, and D. Trentesaux. Dynamic self-or $\mathrm{g}$ ani $\mathrm{z}$ at ion in holonic multi-agent manufacturing systems: The adacor evolution. Computers in Industry 66:99 - 111, 2015.

[13] J. Huang and M. Cakmak. Code3: A system for end-to-end programming of mobile manipulator robots for novices and experts. In Proceedings of the 2017 A CM/IEEE

International Conference on Human-Robot Interaction, HRI '17, pages 453-462, New York, NY, USA, 2017. ACM.

[14] K. Andrews, S. Steinau, V. Künzle, C. Chiao, and M. Reichert. Philharmonic flows - process, humans and information linkage for harmonic business flows, https://www.uni-ulm.de/in/iui-dbis/forschung/laufendeprojekte/philharmonic-flows/, 2019.

[15] M. Bonini. Sechs Makroprozesse der Intralogistik. zafhintralogistik.de, 2019.

[16] M. Crosby, R. Petrick, F. Rovida, and V. Krüger. Integrating mission and task planning in an industrial robotics framework, 2017.

[17] M. Lutz, J. F. Ingles-Romero, D. Stampfer, A. Lotz, C. Vicente-Chicote, and C. Schlegel. Managing variability as a means to promote composability: A robotics perspective. New Perspectives on Information Systems Modeling and Design, pages 274-295, 2018.

[18] M. Onori, H. Alsterman, and J. Barata. An architecture development approach for evolvable assembly systems. In (ISATP 2005). The 6th IEEE International Symposium on Assembly and Task Planning: From Nano to Macro Assembly and Manufacturing, 2005., pages 19-24, July 2005.

[19] R. F. Babiceanu and F. F. Chen. Development and applications of holonic manufacturing systems: A survey. Journal of Intelliqent Manufacturing, 17(1):111-131, Feb 2006.

[20] R. Project. Robmosys wiki - ecosystem organization, https://robmosys.eu/wiki/general_principles:ecosystem:start, 2018.

[21] S. Alexandrova, Z. Tatlock, and M. Cakmak. Roboflow: A flow-based visual programming language for mobile manipulation tasks. In 2015 IEEE International Conference on Robotics and Automation (ICRA), pages 5537-5544, Say $201 \mathrm{~S}$

[22] S. R. R. Center. Intralogistics scenario: Flexible context model adaptation for robotic order picking. https://youtu.be/5116bGhXBr8, 2019.

[23] S. R. R. Center. Intralogistics scenario: Flexible process execution and adaption of a robotic order picking. https://youtu.be/UtO1Dc1B3Cs, 2019.

[24] S. R. R. Center. Smartsoft-robotics. https://sourceforge.net/projects/smart-robotics/.

[25] W. Günthner, J. Durchholz, E. Klenk, and J. Boppert. Schlanke Logistikprozesse - Handbuch für den Planer. Springer, Heidelberg, 2013.

[26] Y. Koren, U. Heisel, E. Jovane, T. Moriwaki, G. Pritschow, G. Ulsoy, and H. Van Brussel. Reconfigurable manufacturing systems. CIRP Annals, N(S):527 - 54S, 19 